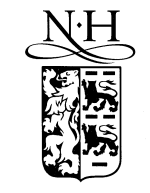

ELSEVIER

Pattern Recognition Letters 19 (1998) 815-820

Pattern Recognition

Letters

\title{
A parallel architecture for implementation of filters based on order statistics
}

\author{
A. Gasteratos, I. Andreadis *, $\mathrm{Ph}$. Tsalides \\ Laboratory of Electronics, Section of Electronics and Information Systems Technology, Department of Electrical and Computer \\ Engineering, Democritus University of Thrace, 67100 Xanthi, Greece
}

Received 30 September 1997; revised 3 April 1998

\begin{abstract}
A new approach for implementing filters based on order statistics is proposed in this letter. As an illustration realisations of range, $\alpha$-trimmed mean and WMMR filters are presented. (c) 1998 Elsevier Science B.V. All rights reserved.

Keywords: Non-linear filters; Order statistics; Computer vision
\end{abstract}

\section{Introduction}

In recent years, significant advances have been made in the development of non-linear image processing methods, since these provide effective solutions to problems where linear techniques are not adequate (Pitas and Venetsanopoulos, 1990). One major category of non-linear filters consist of those based on order statistics (David, 1981). In this category belong the median, rank-order, range, $\alpha$-trimmed mean, weighed majority with minimum range (WMMR), and other popular filters. Filters based on order statistics exhibit excellent robustness properties. For example, they can suppress high frequency and impulse noise, avoiding at the same time exten-

\footnotetext{
* Corresponding author. E-mail: ioannis@ orfeas.ee.duth.gr.
}

sive blurring of the image. They have found numerous applications in digital image analysis, speech processing, coding, etc.

Non-linear filters based on order statistics have been hardware implemented in the past using the threshold decomposition (TD) technique (Fitch et al., 1985; Razi and Chu, 1995) and a bit-serial technique based on Positive Boolean Functions (PBF) (Chen, 1989). These architectures are fixed for a certain type of filter. Also, they can be realised through a sorting network (SN) with compare and swap elements (Pitas and Venetsanopoulos, 1990). A single and efficient structure for any rank order filter realisation based on the majority gate (MG) has been presented by Gasteratos et al. (1997). A comparative study for median filter implementation using $3 \times 3$ pixel image data of 8 -bit resolution is shown in Table 1. In this letter a new real-time parallel architecture for implementing filters based on order statistics is presented. The proposed architecture is recon- 
Table 1

Hardware comparison of existing architectures for median computation using $3 \times 3$-pixel data of 8 -bit resolution

\begin{tabular}{|c|c|c|c|c|}
\hline & $\mathrm{TD}$ & PBF & $\mathrm{SN}$ & MG \\
\hline NOT & - & $8 \times 18$ & $36 \times 24$ & $8 \times 52$ \\
\hline $\mathrm{AND} 2^{\mathrm{a}}$ & - & $8 \times 36$ & $36 \times 17$ & $8 \times 68$ \\
\hline OR2 & - & $8 \times 9$ & - & $8 \times 17$ \\
\hline XNOR2 & - & $8 \times 9$ & - & $8 \times 17$ \\
\hline NOR2 & - & - & $36 \times 8$ & - \\
\hline NAND2 & - & - & $36 \times 24$ & - \\
\hline AND3 & - & - & $36 \times 1$ & - \\
\hline AND4 & - & - & $36 \times 1$ & - \\
\hline AND5 & $256 \times 126$ & $8 \times 126$ & $36 \times 1$ & - \\
\hline AND6 & - & - & $36 \times 1$ & - \\
\hline AND7 & - & - & $36 \times 1$ & - \\
\hline AND8 & - & - & $36 \times 1$ & - \\
\hline OR8 & - & - & $36 \times 1$ & - \\
\hline OR126 & $256 \times 1$ & $8 \times 1$ & - & - \\
\hline $\begin{array}{l}\text { Decoder of } 1 \\
\text { grey-level image } \\
\text { to } 256 \text { binary images }\end{array}$ & 1 & - & - & - \\
\hline $\begin{array}{l}\text { Adder of } 256 \\
\text { 1-bit inputs }\end{array}$ & 1 & - & - & - \\
\hline
\end{tabular}

${ }^{\mathrm{a}}$ The number denotes the inputs: e.g. AND5 is a five input AND gate.

figurable and realisations of range, $\alpha$-trimmed mean and WMMR filters are presented.

\section{Filters based on order statistics}

The input of rank order filters is a data window with an odd number of elements. These elements are sorted in ascending order and the output of the rank order filter with rank $r$ is the $r$-th element ( $r$-th order statistic). Special cases of rank order filters are median, min and max filters.

Range filters are an extension of rank order filters (Bailey and Hodgson, 1985). Let $x_{(1)}, x_{(2)}, \ldots, x_{(N)}$ be the order statistics of the input data window. The output of this filter is

$y=x_{(i)}-x_{(j)}$,

where $1 \leq j<i \leq N$.

$\alpha$-trimmed mean filters are a class of filters with properties varying between two extremes: the running average and the median filter (Razi and Chu (1995)). The output of this filter is

$y=\frac{1}{N-2[\alpha N]} \sum_{i=[\alpha N]+1}^{N-[\alpha N]} x_{(i)}$, where $[\alpha N]$ is the integer part of $\alpha N$ and $0 \leq \alpha \leq$ $1 / 2$.

When $\alpha$ is 0 , the sum includes all inputs and, therefore, the output is the running average. On the contrary, when $\alpha$ is close or equal to $1 / 2$, the output of the filter is the median.

WMMR filters exhibit the same properties in impulse noise rejection and in edge preservation as do the other order statistics filters. Additionally, WMMR filters optimally enhance non-perfect edges (Longbotham and Eberly, 1993). Consider the weights $b_{1}, b_{2}, \ldots, b_{(N+1) / 2}$, such that $b_{j} \geq 0$ and $\sum_{j=1}^{(N+1) / 2} b_{j}=1$. The ranges $x_{(j+(N-1) / 2)}-x_{(j)}, j=$ $1, \ldots,(N+1) / 2$, are calculated and let the minimum be obtained for $j=I_{1}, I_{2}, \ldots, I_{l}$, where $1 \leq l \leq(N+1) / 2$. The output of this filter is

$$
\begin{aligned}
y= & \frac{1}{l}\left[\sum_{j=1}^{(N+1) / 2} b_{j} x_{\left(I_{1}+j-1\right)}+\sum_{j=1}^{(N+1 / 2)} b_{j} x_{\left(I_{2}+j-1\right)}\right. \\
& \left.+\cdots+\sum_{j=1}^{(N+1 / 2)} b_{j} x_{\left(I_{l}+j-1\right)}\right]
\end{aligned}
$$

\section{Implementation of filters based on order statis- tics}

A generalised computing hardware structure of a filter based on order statistics is shown in Fig. 1. The first stage of this structure consists of $N$ order statistic modules (OSM) (Gasteratos et al., 1997), of

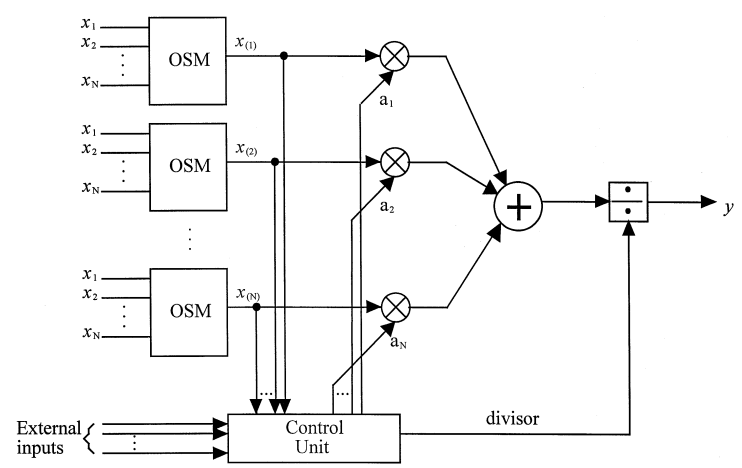

Fig. 1. Generalised computing hardware structure of a filter based on order statistics. 


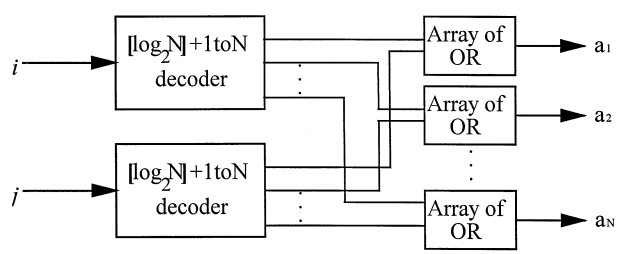

Fig. 2. Control unit for range filtering.

$N$ inputs $\left(x_{1}, x_{2}, \ldots, x_{N}\right)$ each. A different order statistic $x_{(1)}, x_{(2)}, \ldots, x_{(N)}$ is provided by each of these modules. The control unit is associated with the type of the order statistic filter. The inputs to this unit are external inputs (the ranges and factor $\alpha$ in range and $\alpha$-trimmed mean filters, respectively), or the order statistics (WMMR filters). The output of this unit are the divisor and coefficients $a_{1}, a_{2}, \ldots$, $a_{N}$, which are either 0 or 1 in most types of filters. These coefficients are multiplied with the corresponding $x_{(i)}$. In the case of WMMR coefficients, $a_{i}$ are deduced from $b_{j}$, according to Eq. (3). Usually $b_{j}$ are $0.5,0.25,0.125$ or 0 (Longbotham and Eberly, 1993). Multiplication can be performed through shift registers and adders. The shift registers shift right $x_{(i)}$ one place, two places, three places or $n$ places, respectively, where $n$ is the resolution of $x_{(i)}$. Thus, products $b_{j} \cdot x_{(i)}$ are formed and are used as inputs to adders, in order to calculate products $a_{i} \cdot x_{(i)}$. The final stage of the proposed hardware structure is a divider.

The control unit for range filters is shown in Fig. 2 . The external inputs to this unit are numbers $i$ and $j$, which correspond to the order statistics that are subtracted, according to Eq. (1). These numbers are the inputs to two $\left[\log _{2} N\right]+1$ to $N$ decoders. The output of the upper decoder is 1 at position $i$, and 0 elsewhere. The output of the other decoder is -1 at position $j$, and 0 elsewhere. The outputs of the two decoders, combined one-by-one through $N$ arrays of OR gates, produce coefficients $a_{i}$.

The control unit for $\alpha$-trimmed mean filters is a decoder having external input number $\alpha$ and outputs coefficients $a_{i}$. Table 2 contains the truth table of such a decoder for a 9-input data window. The intervals of the values of $\alpha$, the coded values of $\alpha$, which are the inputs to the decoder and coefficients $a_{i}$ are shown in columns one, two and three, respectively. The divisor deduced from factor $\alpha$ is shown in the last column. For a relatively small image data window and pixel resolution, the required division can be efficiently performed through a Look Up Table (LUT). For example, in the usual case of Table 2 and with 8-bit resolution numbers, a LUT of $2304=9 \times 2^{8}$ memory locations is required.

The control unit for WMMR filters is shown in Fig. 3. The ranges $x_{(j+(N-1) / 2)}-x_{(j)}$ are computed by means of $(N+1) / 2$ subtractors. The minimum range is calculated through an $(N+1) / 2$ input OSM. In order to trace the positions $\left(I_{1}, I_{2}, \ldots, I_{l}\right)$ of the minimum range, the ranges and the minimum range are used as inputs to arrays of XOR gates. These produce 0 everywhere, except at the positions of the minimum range, where the outputs are 1 . The outputs of each array of XOR gates are then fed into an OR gate, to produce a single bit output, which determines the positions of the minimum range. The outputs of the latter gates are connected to a combinational circuit with $(N+1) / 2$ inputs and $N$ outputs, corresponding to coefficients $a_{i}$. The outputs of the OR gates are also used as inputs to an adder, which produces the divisor $l$. An illustrative truth table of such a combinational circuit for $N=5$ and $\left\{b_{1}, b_{2}, b_{3}\right\}=\{0.25,0.5,0.25\}$ is shown in Table 3 .

Table 2

Functional table for realisation of $\alpha$-trimmed mean filters

\begin{tabular}{llllllllllll}
\hline$\alpha$ & Coded $\alpha$ & $a_{1}$ & $a_{2}$ & $a_{3}$ & $a_{4}$ & $a_{5}$ & $a_{6}$ & $a_{7}$ & $a_{8}$ & $a_{9}$ & $N-2[\alpha N]$ \\
\hline $0 \leq \alpha<1 / 9$ & 000 & 1 & 1 & 1 & 1 & 1 & 1 & 1 & 1 & 1 & 9 \\
$1 / 9 \leq \alpha<2 / 9$ & 001 & 0 & 1 & 1 & 1 & 1 & 1 & 1 & 1 & 0 & 7 \\
$2 / 9 \leq \alpha<3 / 9$ & 010 & 0 & 0 & 1 & 1 & 1 & 1 & 1 & 0 & 0 & 5 \\
$3 / 9 \leq \alpha<4 / 9$ & 011 & 0 & 0 & 0 & 1 & 1 & 1 & 0 & 0 & 0 & 3 \\
$4 / 9 \leq \alpha<1 / 2$ & 100 & 0 & 0 & 0 & 0 & 1 & 0 & 0 & 0 & 0 & 1 \\
\hline
\end{tabular}




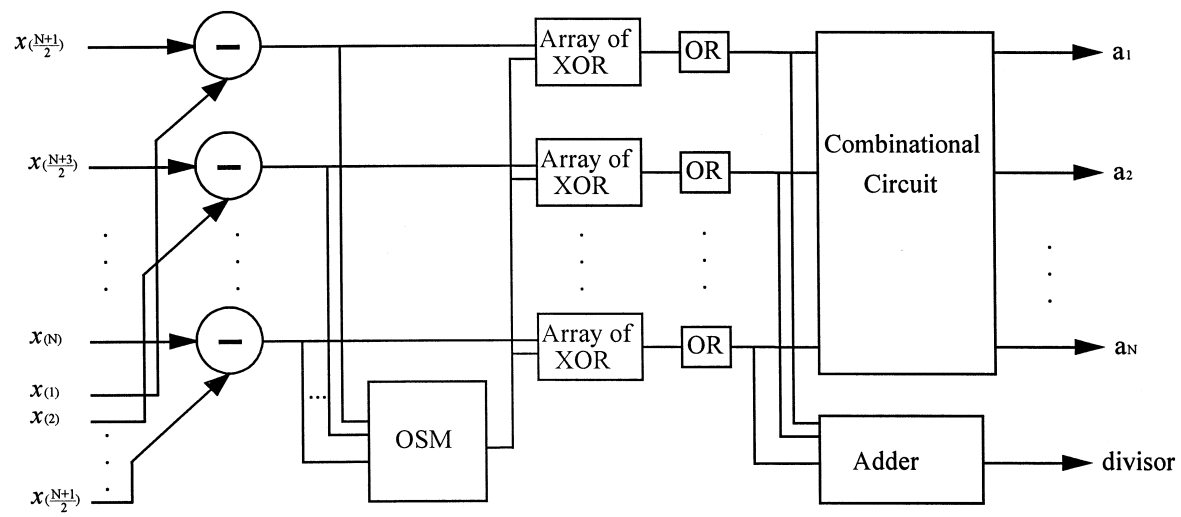

Fig. 3. Control unit for WMMR filtering.

The first three columns are all the possible OR gates outputs, which correspond to the positions of the minimum range. The next five columns are coefficients $a_{i}$, which are computed from coefficients $b_{1}$, $b_{2}$ and $b_{3}$, according to Eq. (3).

Range and $\alpha$-trimmed mean filters can be realised through an SN with compare and swap elements, the TD and the PBF techniques. Table 4 presents an illustrative example for the hardware requirements of the proposed architecture, TD, PBF and SN techniques, for a 5-pixel window and 8-bit pixel resolution. Table 5 shows the number of transistors required for each standard cell mentioned in Table 4. From both Tables 4 and 5 it is obvious that the proposed architecture is superior in terms of silicon area, when compared with the TD technique. Furthermore, in the MG technique, hardware complexity grows linearly both in terms of the number of inputs and resolution of pixels, whereas in the TD technique, it grows exponentially. In the presented example, the PBF and MG techniques are comparable in terms of hardware complexity. However, in the PBF technique, hardware grows as a function of $N$ !; for example, in the median filter this function is $N ! /([N / 2] ![1+N / 2] !)$ (Lee and Jen, 1992). Thus, even for the typical $3 \times 3$-pixel window, the $\mathrm{MG}$ technique becomes advantageous. Both techniques can attain very high operation speeds, the MG technique being slightly faster, due to the presence of an extra multi-input gate level in the PBF implementation. Also, in the presented case, the $\mathrm{SN}$ requires less silicon area than that of the MG technique. Here, hardware complexity is proportional to a factor $N[N / 2]$ (Lee and Jen, 1992). Therefore, for larger size input windows, the $\mathrm{MG}$ technique becomes more attractive than the SN technique. Additionally, the $\mathrm{SN}$ is rather slow when compared with the MG technique. This is due to the fact that the processing element of the MG technique consists of only three levels of gates (Gasteratos et al., 1997), whilst each compare and swap element consists of eight levels of gates (includes a comparator and a multiplexer).

Table 3

An example truth table for the combinational circuit of Fig. $3\left(N=5\right.$ and $\left.\left\{b_{1}, b_{2}, b_{3}\right\}=\{0.25,0.5,0.25\}\right)$

\begin{tabular}{lllllllll}
\hline$x_{(3)}-x_{(1)}$ & $x_{(4)}-x_{(2)}$ & $x_{(5)}-x_{(3)}$ & $a_{1}$ & $a_{2}$ & $a_{3}$ & $a_{4}$ & $a_{5}$ & Divisor \\
\hline 0 & 0 & 1 & 0 & 0 & 0.25 & 0.5 & 0.25 & 1 \\
0 & 1 & 0 & 0 & 0.25 & 0.5 & 0.25 & 0 & 1 \\
0 & 1 & 1 & 0 & 0.25 & 0.75 & 0.75 & 0.25 & 2 \\
1 & 0 & 0 & 0.25 & 0.5 & 0.25 & 0 & 0 & 1 \\
1 & 0 & 1 & 0.25 & 0.5 & 0.5 & 0.5 & 0.25 & 2 \\
1 & 1 & 0 & 0.25 & 0.75 & 0.75 & 0.25 & 0 & 2 \\
1 & 1 & 1 & 0.25 & 0.75 & 1 & 0.75 & 0.25 & 3 \\
\hline
\end{tabular}


Table 4

An illustrative example for the hardware requirements of the TD, PBF, SN and MG techniques, for a 5-pixel window and 8-bit pixel resolution

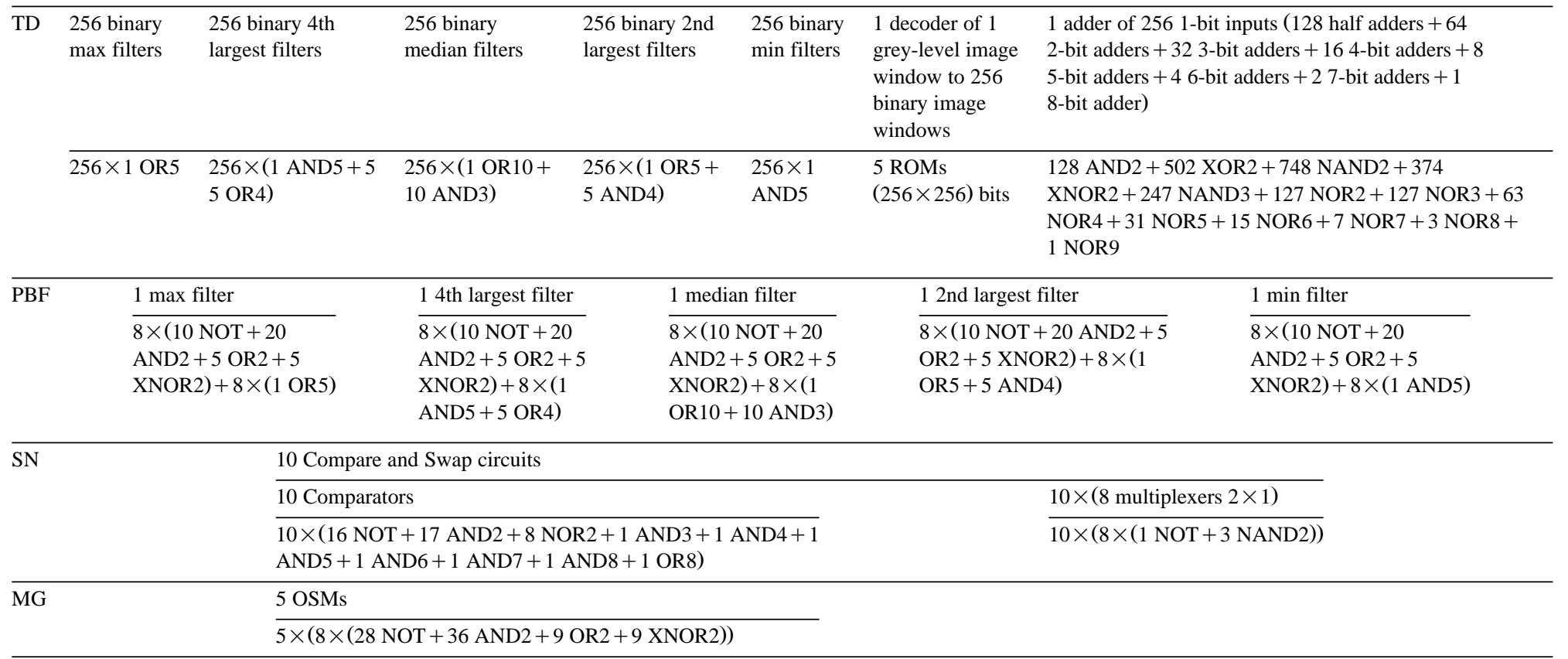


Table 5

Hardware requirements, in terms of transistors (CMOS technology), for each standard cell of Table 4

\begin{tabular}{ll}
\hline $\begin{array}{l}\text { Number of } \\
\text { transistors }\end{array}$ & Standard cell \\
\hline 2 & NOT \\
4 & NAND2, NOR2 \\
6 & AND2, OR2, NAND3, NOR3 \\
8 & AND3, NOR4 \\
10 & AND4, OR4, NOR5 \\
11 & XOR2, XNOR2 \\
12 & AND5, OR5, NOR6 \\
14 & AND6, NOR7 \\
16 & AND7, NOR8 \\
18 & AND8, OR8, NOR9 \\
22 & OR10 \\
\hline
\end{tabular}

Hardware structures for WMMR filters have not been reported in the literature yet.

\section{Conclusions}

A parallel architecture for implementing filters based on order statistics has been presented in this letter. Different filters based on order statistics are obtained, by altering the Control Unit. Efficient realisations of range, $\alpha$-trimmed mean and WMMR filters have been illustrated.

\section{References}

Bailey, D.G., Hodgson, R.M., 1985. Range filters: local-intensity subrange filters and their properties. Image Vision Comp. 3, 99-110.

Chen, K., 1989. Bit-serial realizations of a class of nonlinear filters based on positive boolean functions. IEEE Trans. Circuits Syst. 36, 785-794.

David, H.A., 1981. Order Statistics. Wiley, New York.

Fitch, J.P., Coyle, E.J., Gallagher, N.C., 1985. Threshold decomposition of multidimensional rank order operators. IEEE Trans. Circuits Syst. 32, 445-450.

Gasteratos, A., Andreadis, I., Tsalides, Ph., 1997. Realization of rank-order filters based on majority gate. Pattern Recognition 30, 1571-1576.

Lee, C.L., Jen, C.W., 1992. Bit-sliced median filter design based on majority gate. IEE Proc.-G 139, 63-70.

Longbotham, H., Eberly, D., 1993. The WMMR filters: a class of robust edge enhancers. IEEE Trans. Signal Process. 41, 16801685.

Pitas, I., Venetsanopoulos, A.N., 1990. Nonlinear Digital Filters: Principles and Applications. Kluwer Academic Publishers, Boston.

Razi, D.H., Chu, C.H., 1995. Realization of $\alpha$-trimmed mean filter on the threshold decomposition architecture. Optical Engineering 34, 2767-2771. 